\title{
Anomalous dispersion of longitudinal optical phonons in oxygen-doped La2- xSrxCuO4+
}

Tejsner, Tim; Piovano, Andrea; Tutueanu, Ana; Romer, Astrid T.; Wells, Barrett O.; Grivel, Jean-Claude; Boehm, Martin; Udby, Linda

Published in:

Physical Review B

Link to article, DOI:

10.1103/physrevb.101.100504

Publication date:

2020

Document Version

Publisher's PDF, also known as Version of record

Link back to DTU Orbit

Citation (APA):

Tejsner, T., Piovano, A., Tutueanu, A., Romer, A. T., Wells, B. O., Grivel, J-C., Boehm, M., \& Udby, L. (2020). Anomalous dispersion of longitudinal optical phonons in oxygen-doped $\mathrm{La}_{2-\mathrm{x}} \mathrm{Sr}_{\mathrm{x}} \mathrm{CuO}_{4+}$. Physical Review $B$, 101(10). https://doi.org/10.1103/physrevb.101.100504

\section{General rights}

Copyright and moral rights for the publications made accessible in the public portal are retained by the authors and/or other copyright owners and it is a condition of accessing publications that users recognise and abide by the legal requirements associated with these rights.

- Users may download and print one copy of any publication from the public portal for the purpose of private study or research.

- You may not further distribute the material or use it for any profit-making activity or commercial gain

- You may freely distribute the URL identifying the publication in the public portal 


\title{
Anomalous dispersion of longitudinal optical phonons in oxygen-doped $\mathrm{La}_{2-x} \mathrm{Sr}_{x} \mathrm{CuO}_{4+\delta}$
}

\author{
Tim Tejsner $\odot,{ }^{1,2}$ Andrea Piovano $\odot,{ }^{1}$ Ana Ţuţueanu, ${ }^{1,2}$ Astrid T. Rømer $\odot,{ }^{1}$ Barrett O. Wells, ${ }^{3}$ \\ Jean-Claude Grivel, ${ }^{4}$ Martin Boehm, ${ }^{1}$ and Linda Udby $\odot^{2}$ \\ ${ }^{1}$ Institut Laue-Langevin, 71 Avenue des Martyrs, 38000 Grenoble, France \\ ${ }^{2}$ Nanoscience Center, Niels Bohr Institute, University of Copenhagen, DK-2100 Copenhagen, Denmark \\ ${ }^{3}$ Department of Physics, University of Connecticut, Storrs, Connecticut 06269, USA \\ ${ }^{4}$ Department of Energy Conversion and Storage, Technical University of Denmark, Frederiksborgvej 399, DK-4000 Roskilde, Denmark
}

(Received 25 October 2019; accepted 11 March 2020; published 25 March 2020)

\begin{abstract}
Inelastic neutron scattering has been used to study the in-plane $\mathrm{Cu}-\mathrm{O}$ bond-stretching mode in oxygendoped $\mathrm{La}_{1.94} \mathrm{Sr}_{0.06} \mathrm{CuO}_{4.035}\left(T_{c}=38 \mathrm{~K}\right)$ and $\mathrm{La}_{2} \mathrm{CuO}_{4+\delta}\left(T_{c}=43 \mathrm{~K}\right)$. Similar to results from optimally doped $\mathrm{La}_{1.85} \mathrm{Sr}_{0.15} \mathrm{CuO}_{4}\left(T_{c}=35 \mathrm{~K}\right)$, we observe anomalous features in the dispersion of this half-breathing mode in the form of a softening halfway through the Brillouin zone. Considering the differences in electronic structure and local environment between the oxygen- and strontium-doped compounds with similar $T_{\mathrm{c}}$, we rule out a connection between the phonon anomaly and structural instabilities related to the specific dopant type. We interpret the phonon anomaly as a signature of correlated charge fluctuations ubiquitous in optimally doped superconductors.
\end{abstract}

DOI: 10.1103/PhysRevB.101.100504

A widely accepted description of the low-temperature electronic state of underdoped cuprate superconductors is the "stripe" picture, where, due to hole doping, the antiferromagnetic (AFM) ground state of the parent compound is segregated by channels of charge resulting in magnetic antiphase boundaries [1]. Experimentally, the magnetic part of the stripes shows up in neutron-scattering experiments as a modulated AFM structure (static magnetic stripes) and excitations emerging with similar wave vectors, in the low-energy regime (dynamic magnetic stripes).

While experimental evidence of magnetic stripes has been extensively documented (see, e.g., [2] for a review), the charge component of the stripes is more elusive. Static charge stripes only show up in superconducting (SC) samples close to the $x=\frac{1}{8}$ anomaly [1,3-6] and evidence for dynamic charge stripes has been reported for $\mathrm{La}_{2-x} \mathrm{Sr}_{x} \mathrm{CuO}_{4}[7,8]$, $\mathrm{YBa}_{2} \mathrm{Cu}_{3} \mathrm{O}_{7-\delta}$ [9], and $\mathrm{La}_{2-x} \mathrm{Sr}_{x} \mathrm{NiO}_{4}$ [10]. Understanding the relationship between these signatures of stripe formation as a function of doping would be a crucial leap forward in our understanding of the cuprates.

Recently, it was discovered that the dispersion of the $\mathrm{Cu}-\mathrm{O}$ bond-stretching longitudinal-optical (LO) phonon in $\mathrm{SC} \mathrm{La}_{1.85} \mathrm{Sr}_{0.15} \mathrm{CuO}_{4}\left(\mathrm{LSCO} 15, T_{\mathrm{c}}=38 \mathrm{~K}\right)$ [11] and $\mathrm{La}_{1.80} \mathrm{Sr}_{0.20} \mathrm{CuO}_{4} \quad\left(T_{\mathrm{c}} \approx 35 \mathrm{~K}\right) \quad$ [12] displays a strong anomalous softening interpreted as a coupling to a novel charge collective mode [12]. Furthermore, merely a weak signature of the anomaly is visible in the phonon linewidth of $\mathrm{La}_{1.93} \mathrm{Sr}_{0.07} \mathrm{CuO}_{4} \quad\left(T_{\mathrm{c}} \approx 15 \mathrm{~K}\right)$ and $\mathrm{La}_{1.75} \mathrm{Sr}_{0.25} \mathrm{CuO}_{4}$ ( $\left.T_{\mathrm{c}} \approx 15 \mathrm{~K}\right)$, suggesting that the strength of the anomaly tracks the doping dependence of $T_{c}$ [12]. Similar phonon anomalies have been observed in $\mathrm{Bi}_{2} \mathrm{Sr}_{2} \mathrm{CaCu}_{2} \mathrm{O}_{8+\delta}$ [13], $\mathrm{La}_{1.875} \mathrm{Ba}_{0.125} \mathrm{CuO}_{4} \quad(\mathrm{LBCO}) \quad[14], \quad \mathrm{La}_{1.48} \mathrm{Nd}_{0.4} \mathrm{Sr}_{0.12} \mathrm{CuO}_{4}$ (LNSCO) [11], and $\mathrm{YBa}_{2} \mathrm{Cu}_{3} \mathrm{O}_{6.6}$ [15] hinting at a ubiquitous feature of cuprate superconductors.
In order to further investigate the robustness of the phonon anomaly in the $0.1<x<0.2$ doping range, we examine two compounds derived from $\mathrm{La}_{2} \mathrm{CuO}_{4}$ by two distinct doping procedures giving rise to unique magnetic, structural, and superconducting properties.

Hole doping of the parent compound $\mathrm{La}_{2} \mathrm{CuO}_{4}$ can be performed by introducing two distinct dopant species. Replacing $\mathrm{La}^{3+}$ for $\mathrm{Sr}^{2+}$ yields $\mathrm{La}_{2-x} \mathrm{Sr}_{x} \mathrm{CuO}_{4}$ (LSCO) with "quenched doping" [16], meaning that Sr has a fixed random distribution on La sites after crystal growth. On the other hand, an "annealed doping" [16] can be obtained by introducing excess, mobile oxygen anions into the lattice by electrochemical methods [17], obtaining $\mathrm{La}_{2} \mathrm{CuO}_{4+\delta}$ (LCO+O). At low $\mathrm{Sr}$ concentrations $(x \leqslant 0.14)$ it is possible to combine $\mathrm{Sr}$ and $\mathrm{O}$ dopants, resulting in "codoped" $\mathrm{La}_{2-x} \mathrm{Sr}_{x} \mathrm{CuO}_{4+\delta}$ (LSCO+O) [18]. Figure 1(a) depicts the crystallographic sites of O/Sr dopant ions [19-21].

Quenched $\mathrm{Sr}^{2+}$ doping creates a superconductor where $T_{\mathrm{c}}$ varies continuously with doping, forming the so-called superconducting dome for $0.05 \leqslant x \leqslant 0.25$. Meanwhile the mobile $\mathrm{O}$ dopants only seem to allow for certain superconducting phases to emerge $\left(T_{\mathrm{c}} \approx 15,30,40 \mathrm{~K}\right)$ due to oxygen content [22], pressure [23], or thermal treatment [24].

At sufficient $\mathrm{O}$ doping, $\mathrm{LSCO}+\mathrm{O}$ is superconducting at ambient pressure below $T_{\mathrm{c}} \approx 40 \mathrm{~K}$, regardless of $\mathrm{Sr}$ content [18]. While the underlying mechanism for the connection between annealed disorder and superconductivity is far from settled, the distribution of $\mathrm{O}$ dopants is clearly distinct from the distribution of $\mathrm{Sr}^{2+}$ dopants. Contrary to the quenched $\mathrm{Sr}^{2+}$ dopants, annealed O dopants in LSCO+O are responsible for a number of structural ordering phenomena such as staging [25,26] and fractal-like distributions of ordered superstructure patches [24,27].

Differences can also be observed in the magnetic structure of the two compounds. In $\mathrm{LSCO}+\mathrm{O}$, the transition to static 


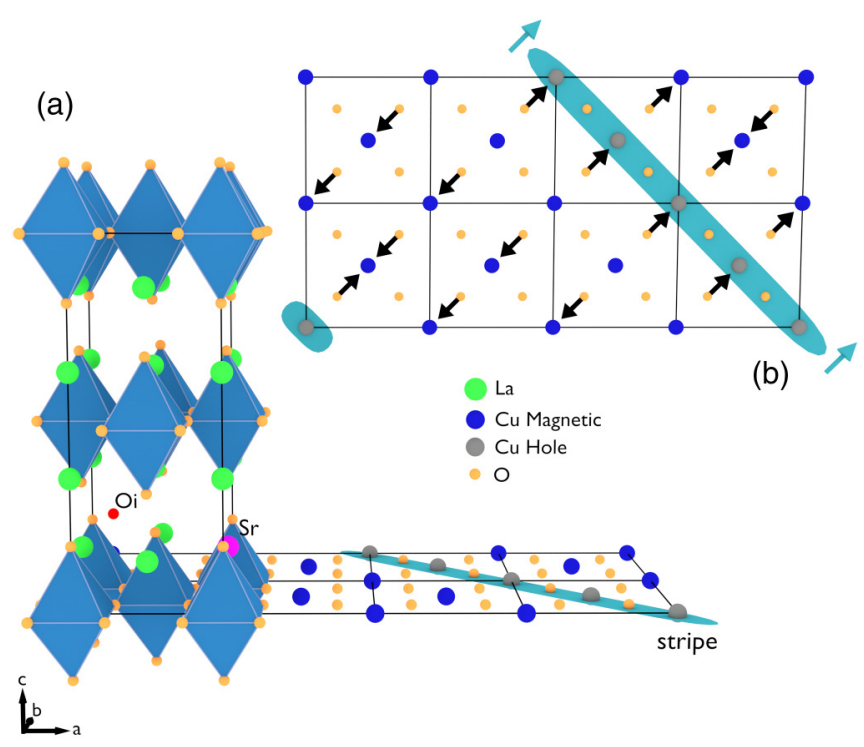

FIG. 1. (a) Sketch of $\mathrm{La}_{2-x} \mathrm{Sr}_{x} \mathrm{CuO}_{4+\delta}$ in the orthorhombic unit cell with two distinct dopant species: $\mathrm{Sr}^{2+}$ replacing $\mathrm{La}^{3+}$ and interstitial oxygen (Oi). The depicted supercell contains 32 formula units (eight orthorhombic conventional cells), and the two singular dopant species thus represent a doping of $n_{\mathrm{h}}=\frac{1+2}{32} \approx 0.09$. Blue $\mathrm{Cu}$ atoms represent antiferromagnetic regions while gray $\mathrm{Cu}$ atoms represent a charge stripe. (b) Sketch of in-phase displacement of dynamic charge domain wall modes coupled to the phonon. Black arrows represent the displacement due to the $\mathrm{Cu}-\mathrm{O}$ half-breathing mode at $\boldsymbol{q}=(0.25,0.25,0)$, while teal arrows represent the in-phase mode of the charge stripe [28].

magnetic stripe order, $T_{\mathrm{N}}$, roughly coincides with $T_{\mathrm{c}}$ [29]. In LSCO, on the other hand, it generally holds that the spins freeze gradually with decreasing temperature and $T_{\mathrm{N}}<T_{\mathrm{c}}$ up to a doping of $x=0.13$, above which the magnetic spectrum becomes gapped [30,31]. This difference in magnetic signatures can partially be explained by the fact that $\mathrm{LSCO}+\mathrm{O}$ phase separates into distinct spin-stripe-ordered $\left(x \approx \frac{1}{8}\right)$ and optimally superconducting $(x \approx 0.16)$ phases, regardless of strontium content prior to oxygenation [18,29].

In this Rapid Communication, we investigate the effects of oxygen disorder on the $\mathrm{Cu}-\mathrm{O}$ bond-stretching phonon mode by providing evidence of phonon anomalies in oxygen-doped $\mathrm{LCO}+\mathrm{O}$ and codoped $\mathrm{La}_{1.94} \mathrm{Sr}_{0.06} \mathrm{CuO}_{4.035}$ (LSCO6+O). If the phonon anomaly is related to a specific structural instability due to quenched disorder, one would expect a different response of the lattice in oxygen-doped compounds with annealed disorder. Our results show remarkable similarities of the phonon anomaly in $\mathrm{LCO}+\mathrm{O}$, $\mathrm{LSCO}+\mathrm{O}$, and LSCO. This points towards a purely electronic origin of the phonon anomaly. In order to investigate the possible connection to static stripe order, we explore the effects of a $10 \mathrm{~T}$ applied magnetic field. A $10 \mathrm{~T}$ field is known to significantly increase the static magnetic response for both $\mathrm{LCO}+\mathrm{O}$ [32] and $\mathrm{LSCO6}+\mathrm{O}$ [33]. The presence of a field-induced enhancement of the phonon anomaly would indicate a direct connection to static magnetic stripe order, while no straightforward link can be deduced in the absence of a field effect. We observe no detectable change in the phonon signal with applied field, an observation which is compatible with the phonon coupling to dynamic charge fluctuations as has been suggested previously [14]. Our observations thus reinforce and expand this interpretation more broadly for doped cuprates.

The samples are high-quality $\mathrm{LCO}+\mathrm{O}$ and $\mathrm{LSCO} 6+\mathrm{O}$ single crystals. The oxygen-stoichiometric samples were grown by the traveling solvent float zone method. Subsequently, oxygen intercalation was performed using wet-chemical methods [17]. Both samples are optimally superconducting with $T_{\mathrm{c}}=43 \mathrm{~K}(\mathrm{LCO}+\mathrm{O})$ and $T_{\mathrm{c}}=37.5 \mathrm{~K}(\mathrm{LSCO}+\mathrm{O})$, confirmed by magnetization measurements (see Fig. S1 in the Supplemental Material (SM) [34]). Prior to oxygen intercalation the samples were insulating (LCO) or had $T_{\mathrm{c}}^{\text {onset }}=8 \mathrm{~K}$ (LSCO6).

Neutron-scattering experiments were performed on the IN8 thermal triple-axis spectrometer at Institut Laue-Langevin, Grenoble [35,36]. The instrument was configured with a silicon (311) monochromator and pyrolythic graphite (002) analyzer in order to access the desired dynamical range while obtaining the best compromise between neutron intensity and energy resolution $(\approx 4-5 \mathrm{meV}$ at $80 \mathrm{meV}$ energy transfer with final wave vector $k_{\mathrm{f}}=2.226 \AA^{-1}$ ). We made use of a position sensitive detector with a horizontal spatial resolution of $5 \mathrm{~mm}$ in order to identify and subtract spurious scattering (see Figs. S3 and S4 in the SM [34]). Throughout this Rapid Communication, all Miller indices are described with reference to the orthorhombic Bmab space group and the samples are aligned in the $a-b$ plane. Lattice parameters were found to be $a=b \approx 5.35(5) \AA, c \approx 13.1(1) \AA$ for both samples.

Figure 2 shows the reduced data at selected wave vectors for both $\mathrm{LSCO} 6+\mathrm{O}$ and $\mathrm{LCO}+\mathrm{O}$. The data has been fitted to a damped harmonic oscillator (DHO) model [37] with a flat background, convoluted with instrument resolution:

$$
S(\boldsymbol{q}, \omega)=I_{\mathrm{ph}} \frac{1}{\pi \omega_{\boldsymbol{q}}} \frac{\gamma}{\left(\omega-\omega_{\boldsymbol{q}}\right)^{2}+\gamma^{2}}+I_{\mathrm{BG}},
$$

where $I_{\mathrm{ph}}$ is the phonon intensity, $\omega_{q}$ the phonon energy at wave vector $\boldsymbol{q}, \gamma$ the phonon linewidth, and $I_{\mathrm{BG}}$ the background intensity.

The extracted dispersion from the zero-field data is shown in Fig. 3 along with a normal sinusoidal dispersion, $\hbar \omega_{q}=$ $\alpha \cos (2 \pi q)+\beta$, inferred from phonon calculations on LSCO using density functional theory (see Fig. S5 in the SM [34] and Ref. [38]). We fit the cosine function to points near the zone center $[\boldsymbol{q}=(0,0,0)]$ and edge $\left[\boldsymbol{q}=\left(\frac{1}{2}, \frac{1}{2}, 0\right)\right]$ to obtain the dashed curves of Fig. 3(a). To quantify the magnitude of the anomaly, we define the "anomaly signal" as the difference between the normal dispersion and the measured data [gray shaded area in Fig. 3(a)]. Figure 3(b) shows our anomaly signal for $\mathrm{LCO}+\mathrm{O}$ and $\mathrm{LSCO} 6+\mathrm{O}$ along with previous results from optimally doped LSCO15 and insulating, stripe-ordered LNSCO [11]. We emphasize the presence of similar anomaly signals on an absolute scale across all studied samples. The linewidth broadening $\gamma$ follows the softening of $\omega_{q}$ similar to what was observed in LSCO15 and LNSCO [11] (see Fig. S2 in the SM [34]). Finally, our data can be described by a linear combination of stripe-ordered and optimally superconducting anomaly signals (see Fig. S7 in the SM [34]), consistent with the observation of phase separation in the (super)oxygenated compounds [18]. 


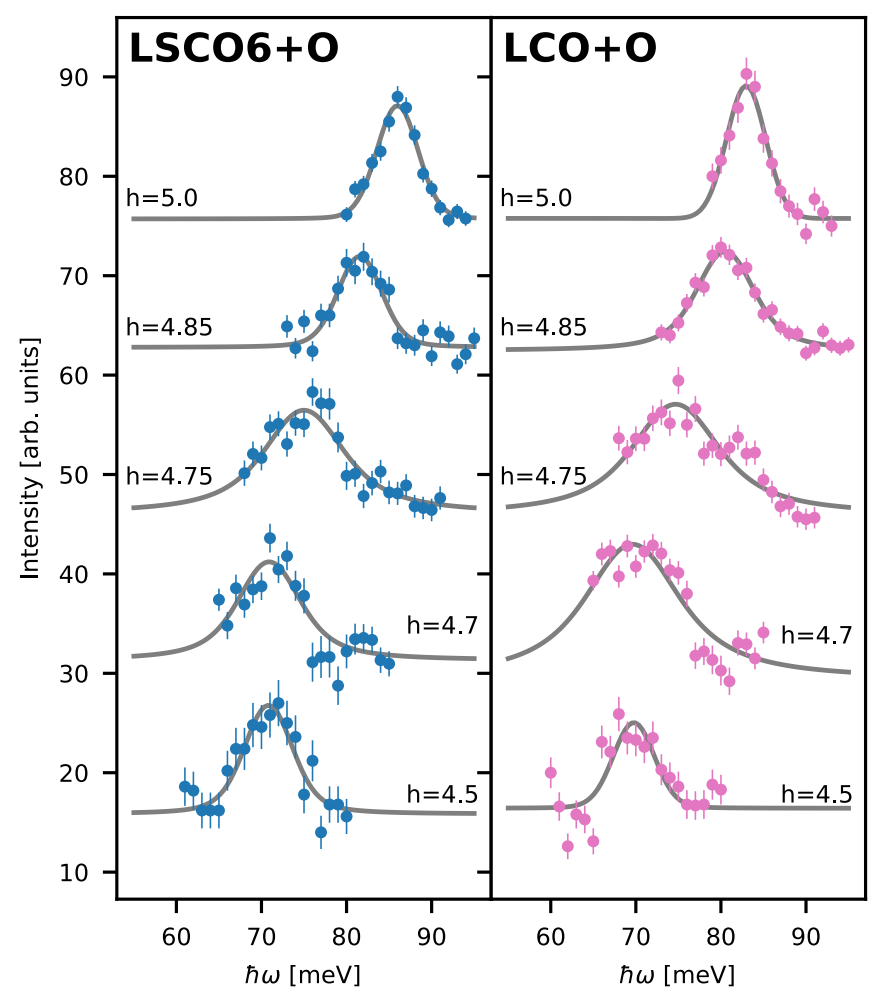

FIG. 2. Reduced data at selected wave vectors of the form $Q=$ $(h, h, 0)$ for both LSCO6+O and LCO+O at $T=5 \mathrm{~K}$ and $H=0 \mathrm{~T}$. Data at $Q=(5,5,0)$ and $Q=(4.85,4.85,0)$ was scaled by a factor of $\frac{1}{2}$ for clarity due to an increase of intensity from the phonon form factor. Data at different $h$ are offset for clarity. Solid lines are fits to a DHO line shape (see text).

To begin the discussion of our results, we remark that softening and/or broadening of phonon modes is generally a signature of an incipient structural or electronic instability. Typical examples include structural phase transitions, $\boldsymbol{q}$ dependence of the electron-phonon matrix element, Fermi surface nesting, and electronic correlations [39]. In order to determine the origin of a given phonon anomaly, it is therefore important to carefully exhaust alternatives before making statements about the connection to novel phases such as dynamic charge stripes [39].

The phonon anomaly appears in the vicinity of the wave vector $\boldsymbol{q}_{\mathrm{co}}=\left(\frac{1}{4}, \frac{1}{4}, 0\right)$, consistent with charge-stripe ordering as illustrated in Fig. 1. Measurements of LNSCO [40], LSCO15 [11], and LBCO [11] have shown a suppression of the anomaly as one moves away from the bond-stretching direction [40], supporting a one-dimensional stripelike picture. Additionally, the phonon anomaly in LSCO15 and LBCO has almost no temperature dependence apart from a slightly sharper peak shape when heating from 10 to $300 \mathrm{~K}[11,14]$. These phenomena rule out anharmonicity and structural inhomogeneity as mechanisms for the phonon anomaly in these systems.

A combination of inelastic x-ray and angle-resolved photoemission spectroscopy (ARPES) measurements on overdoped LSCO ( $x=0.2$ and $x=0.3)$ have shown that the phonon anomaly wave vector is inconsistent with Fermi surface nesting $[12,41]$, contradicting the idea of a phonon

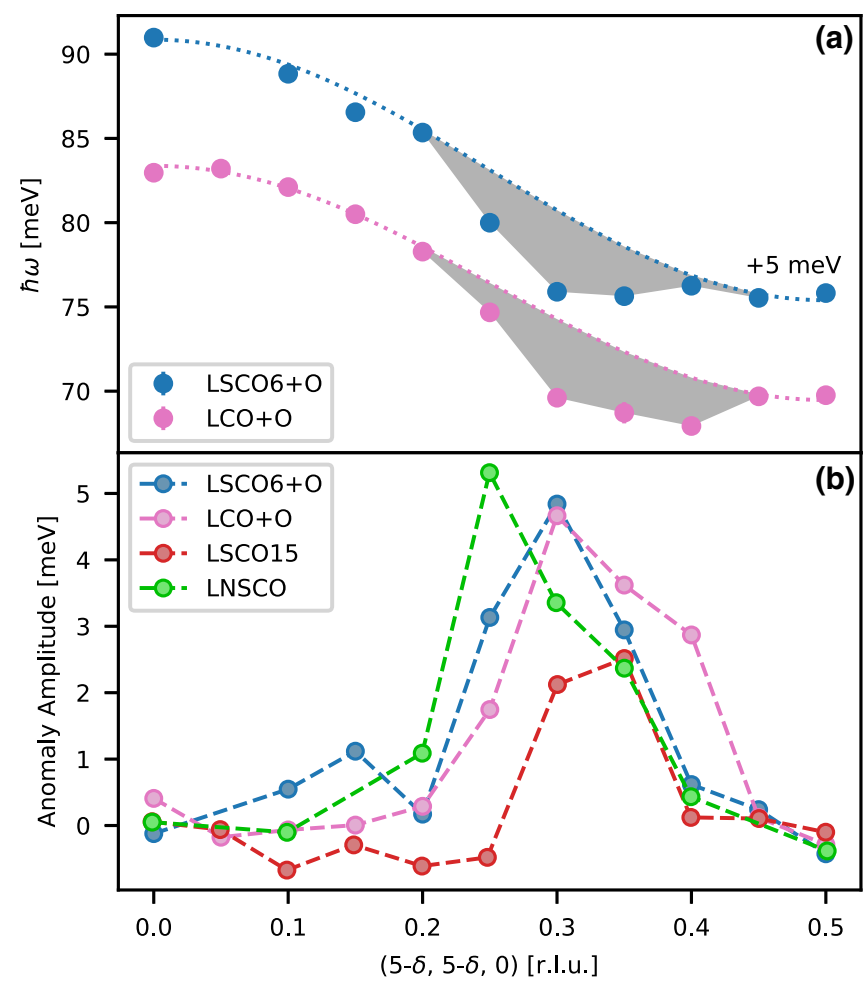

FIG. 3. (a) Dispersion of the LO phonon obtained from the peak positions of individual spectra of both $\mathrm{LCO}+\mathrm{O}$ and $\mathrm{LSCO}+\mathrm{O}$ (offset by $5 \mathrm{meV}$ for clarity). Error bars smaller than the markers are not shown. Dashed line is the normal sinusoidal dispersion as described in the text. All data was obtained at $T=5 \mathrm{~K}$. (b) Difference between sinusoidal and measured dispersion in $\mathrm{La}_{2} \mathrm{CuO}_{4+\delta}(\mathrm{LCO}+\mathrm{O})$, $\mathrm{La}_{2-x} \mathrm{Sr}_{x} \mathrm{CuO}_{4+\delta} \quad(\mathrm{LSCO}+\mathrm{O}), \mathrm{La}_{1.85} \mathrm{Sr}_{0.15} \mathrm{CuO}_{4}$ (LSCO15), and $\mathrm{La}_{1.48} \mathrm{Nd}_{0.4} \mathrm{Sr}_{0.12} \mathrm{CuO}_{4}$ (LNSCO). Data for LSCO15 and LNSCO adapted from [11].

softening due to a Kohn anomaly. A different, possibly $\boldsymbol{q}$ dependent, electron-phonon coupling could still be responsible for the phonon anomaly. Such an effect would renormalize the electronic quasiparticle dispersion (the so-called "ARPES kink" [42]) at energies similar to the phonon softening. The ARPES kink has been observed in LSCO $x=0.2$ and $x=$ 0.3 , but since only LSCO $x=0.2$ shows anomalous phonons, the two phenomena appear to not be connected [12].

Thus, all previous studies are unable to explain the phonon anomaly through conventional means and any coupling to stripe order is likely dynamic. One possible scenario is a coupling of the $\mathrm{Cu}-\mathrm{O}$ bond-stretching phonon with steeply dispersing charge fluctuations. Kaneshita et al. performed calculations based on the Hubbard model of this scenario, predicting anomalous phonon dispersions due to both transverse (meandering) and longitudinal (compression) coherent stripe fluctuations [28] (see Fig. 1 for a sketch of the transverse mode). We emphasize that the observed phonon anomaly reported here (see Fig. 3) and in LBCO/LSCO [11,14] is remarkably similar to the calculation by Kaneshita et al. (see Fig. 5 in Ref. [28]).

Despite differences in the magnetic excitation spectra as recorded by neutron scattering (including low- and zeroenergy transfers), the three materials $\mathrm{LCO}+\mathrm{O}, \mathrm{LSCO} 6+\mathrm{O}$, 


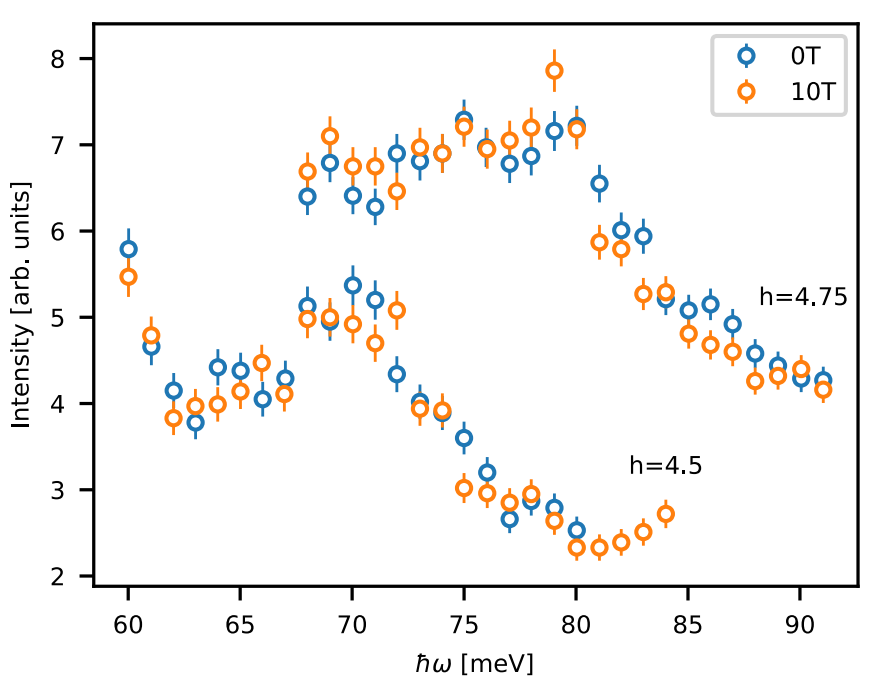

FIG. 4. Comparison of representative constant- $Q(h, h, 0)$ scans of $\mathrm{La}_{1.94} \mathrm{Sr}_{0.06} \mathrm{CuO}_{4.035}$ in zero field and with an applied field of $10 \mathrm{~T}$. All measurements performed at $T=5 \mathrm{~K}$.

and LSCO15 have remarkably similar in-plane $\mathrm{Cu}-\mathrm{O}$ bondstretching dispersions (Fig. 3). Furthermore, static charge order at zero field has been observed in a different sample of $\mathrm{LCO}+\mathrm{O}$ [43] and in LNSCO [3] but so far not in LSCO6+O nor in LSCO15. These observations together rule out a unique, direct connection between static stripes (spin and charge) and the phonon anomaly. In order to further confirm this point, we performed scans of LSCO6+O at selected wave vectors in a $H=10 \mathrm{~T}$ magnetic field which is known to induce a considerable volume of stripelike magnetic order in this particular sample [33]. While static charge order has not been observed in $\mathrm{LSCO} 6+\mathrm{O}$, measurements on LSCO $(x=0.12)$ have shown that static charge and spin stripes respond identically to magnetic fields [4].

Figure 4 contains data at two wave vectors with and without an applied magnetic field of $10 \mathrm{~T}$, clearly showing the absence of any detectable field effect on the in-plane $\mathrm{Cu}-\mathrm{O}$ bond-stretching phonon at $\boldsymbol{q}=\left(\frac{1}{4}, \frac{1}{4}, 0\right)$. These measurements were performed simultaneously with measurements of the low-energy magnetic fluctuations [44], confirming a significant increase in the magnetic spectral weight towards lower energies consistent with the appearance of field-induced stripe order [33]. Thus, the appearance of static magnetic stripe order does not affect the phonon anomaly in LSCO6+O. A similar insensitivity of the phonon anomaly to an applied magnetic field has been observed in underdoped $\left(T_{\mathrm{c}}=66 \mathrm{~K}\right)$ $\mathrm{YBa}_{2} \mathrm{CuO}_{6.6}$ [45].

We have shown that the phonon anomaly is a robust feature in optimally doped as well as stripe-ordered cuprates which is independent of the structural details related to the doping process. Since it is equally well formed in stripe-ordered and optimally doped systems, where the latter show no static magnetic order, the anomaly is surprisingly insensitive to low-energy magnetic characteristics. This is further confirmed by the absence of a magnetic field effect in $\mathrm{LSCO} 6+\mathrm{O}$ which introduces static magnetic stripe order.

The phonon anomaly is strongest in the doping region around optimal $T_{\mathrm{c}}\left(0.125 \leqslant n_{\mathrm{h}} \leqslant 0.20\right)$ (LSCO15, LNSCO [11], LBCO [14], LSCO6+O, LCO+O), regardless of the presence of static charge order (LNSCO [1], LBCO [46]), suppression of bulk superconductivity (LNSCO [3]) or dopant disorder ( $\mathrm{LCO}+\mathrm{O}, \mathrm{LSCO}+\mathrm{O}$ ). In addition, the phonon anomaly is unaffected by magnetic fields (LSCO6+O, $\mathrm{YBa}_{2} \mathrm{CuO}_{6.6}$ [45]) and temperature (LBCO, LSCO15) [11]).

Thus it appears to be an intrinsic, robust signature of doped cuprates near optimal doping. We point out that the phonon anomaly region coincides with the dome of dynamic charge fluctuations recently reported in Ref. [9]. This points to fluctuating charge stripes as the fundamental degrees of freedom in the cuprates and thereby lends credibility to the "electronic liquid crystal" model [47].

In conclusion, we have measured the in-plane $\mathrm{Cu}-\mathrm{O}$ bondstretching phonon in $\mathrm{LSCO}+\mathrm{O}$ and $\mathrm{LCO}+\mathrm{O}$ and provided evidence for significant anomalous behavior. Since one sample $(\mathrm{LCO}+\mathrm{O})$ exhibits charge order [43] while the other (LSCO6+O) does not [33], and since the samples also have different magnetic spectra with distinct field dependencies $[33,48]$ we conclude that the phonon anomaly has no direct, trivial relationship to either magnetic or charge static order. In addition, the unique structural characteristics of oxygen-doped samples rule out a connection between the specific dopant species and the phonon anomaly. We proceed to conclude that the phonon anomaly is a signature of charge stripe fluctuations, which is a common characteristic of the cuprate family and appears to be a prerequisite to optimal superconductivity in these systems.

We acknowledge useful discussions with Kim Lefmann and Henrik Jacobsen. A.T.R. acknowledges support from the Carlsberg Foundation. We would like to thank the ILL for the provision of beam time on IN8 [35,36].
[1] J. M. Tranquada, B. J. Sternlieb, J. D. Axe, Y. Nakamura, and S. Uchida, Nature (London) 375, 561 (1995).

[2] J. M. Tranquada, Spins, Stripes, and Superconductivity in HoleDoped Cuprates, AIP Conf. Proc. No. 1550 (AIP, New York, 2013), p. 114.

[3] J. M. Tranquada, J. D. Axe, N. Ichikawa, Y. Nakamura, S. Uchida, and B. Nachumi, Phys. Rev. B 54, 7489 (1996).
[4] N. B. Christensen, J. Chang, J. Larsen, M. Fujita, M. Oda, M. Ido, N. Momono, E. M. Forgan, A. T. Holmes, J. Mesot, M. Huecker, and M. v Zimmermann, arXiv:1404.3192.

[5] V. Thampy, M. P. M. Dean, N. B. Christensen, L. Steinke, Z. Islam, M. Oda, M. Ido, N. Momono, S. B. Wilkins, and J. P. Hill, Phys. Rev. B 90, 100510(R) (2014).

[6] T. P. Croft, C. Lester, M. S. Senn, A. Bombardi, and S. M. Hayden, Phys. Rev. B 89, 224513 (2014). 
[7] H. Miao, J. Lorenzana, G. Seibold, Y. Y. Peng, A. Amorese, F. Yakhou-Harris, K. Kummer, N. B. Brookes, R. M. Konik, V. Thampy, G. D. Gu, G. Ghiringhelli, L. Braicovich, and M. P. M. Dean, Proc. Natl. Acad. Sci. USA 114, 12430 (2017).

[8] H. Miao, R. Fumagalli, M. Rossi, J. Lorenzana, G. Seibold, F. Yakhou-Harris, K. Kummer, N. B. Brookes, G. D. Gu, L. Braicovich, G. Ghiringhelli, and M. P. M. Dean, Phys. Rev. X 9, 031042 (2019).

[9] R. Arpaia, S. Caprara, R. Fumagalli, G. D. Vecchi, Y. Y. Peng, E. Andersson, D. Betto, G. M. D. Luca, N. B. Brookes, F. Lombardi, M. Salluzzo, L. Braicovich, C. D. Castro, M. Grilli, and G. Ghiringhelli, Science 365, 906 (2019).

[10] S. Anissimova, D. Parshall, G. D. Gu, K. Marty, M. D. Lumsden, S. Chi, J. A. Fernandez-Baca, D. L. Abernathy, D. Lamago, J. M. Tranquada, and D. Reznik, Nat. Commun. 5, 3467 (2014).

[11] D. Reznik, L. Pintschovius, M. Fujita, K. Yamada, G. D. $\mathrm{Gu}$, and J. M. Tranquada, J. Low Temp. Phys. 147, 353 (2007).

[12] S. R. Park, T. Fukuda, A. Hamann, D. Lamago, L. Pintschovius, M. Fujita, K. Yamada, and D. Reznik, Phys. Rev. B 89, 020506(R) (2014).

[13] L. Chaix, G. Ghiringhelli, Y. Y. Peng, M. Hashimoto, B. Moritz, K. Kummer, N. B. Brookes, Y. He, S. Chen, S. Ishida, Y. Yoshida, H. Eisaki, M. Salluzzo, L. Braicovich, Z.-X. Shen, T. P. Devereaux, and W.-S. Lee, Nat. Phys. 13, 952 (2017).

[14] D. Reznik, L. Pintschovius, M. Ito, S. Iikubo, M. Sato, H. Goka, M. Fujita, K. Yamada, G. D. Gu, and J. M. Tranquada, Nature (London) 440, 1170 (2006).

[15] M. Le Tacon, A. Bosak, S. M. Souliou, G. Dellea, T. Loew, R. Heid, K.-P. Bohnen, G. Ghiringhelli, M. Krisch, and B. Keimer, Nat. Phys. 10, 52 (2014).

[16] B. O. Wells, Y. S. Lee, M. A. Kastner, R. J. Christianson, R. J. Birgeneau, K. Yamada, Y. Endoh, and G. Shirane, Science 277, 1067 (1997).

[17] P. Blakeslee, R. J. Birgeneau, F. C. Chou, R. Christianson, M. A. Kastner, Y. S. Lee, and B. O. Wells, Phys. Rev. B 57, 13915 (1998).

[18] H. E. Mohottala, B. O. Wells, J. I. Budnick, W. A. Hines, C. Niedermayer, L. Udby, C. Bernhard, A. R. Moodenbaugh, and F.-C. Chou, Nat. Mater. 5, 377 (2006).

[19] P. G. Radaelli, D. G. Hinks, A. W. Mitchell, B. A. Hunter, J. L. Wagner, B. Dabrowski, K. G. Vandervoort, H. K. Viswanathan, and J. D. Jorgensen, Phys. Rev. B 49, 4163 (1994).

[20] C. Rial, E. Morán, M. A. Alario-Franco, U. Amador, and N. H. Andersen, Phys. C (Amsterdam, Neth.) 254, 233 (1995).

[21] C. Rial, E. Morán, M. A. Alario-Franco, U. Amador, and N. H. Andersen, Phys. C (Amsterdam, Neth.) 278, 122 (1997).

[22] L. H. Liu, G. C. Che, J. Zhao, and Z. X. Zhao, Phys. C (Amsterdam, Neth.) 425, 37 (2005).

[23] B. Lorenz, Z. G. Li, T. Honma, and P.-H. Hor, Phys. Rev. B 65, 144522 (2002).

[24] M. Fratini, N. Poccia, A. Ricci, G. Campi, M. Burghammer, G. Aeppli, and A. Bianconi, Nature (London) 466, 841 (2010).

[25] B. O. Wells, R. J. Birgeneau, F. C. Chou, Y. Endoh, D. C. Johnston, M. A. Kastner, Y. S. Lee, G. Shirane, J. M.
Tranquada, and K. Yamada, Z. Phys. B: Condens. Matter 100, 535 (1996).

[26] P. J. Ray, N. H. Andersen, T. B. S. Jensen, H. E. Mohottala, C. Niedermayer, K. Lefmann, B. O. Wells, M. v. Zimmermann, and L. Udby, Phys. Rev. B 96, 174106 (2017).

[27] N. Poccia, A. Ricci, G. Campi, M. Fratini, A. Puri, D. D. Gioacchino, A. Marcelli, M. Reynolds, M. Burghammer, N. L. Saini, G. Aeppli, and A. Bianconi, Proc. Natl. Acad. Sci. USA 109, 15685 (2012).

[28] E. Kaneshita, M. Ichioka, and K. Machida, Phys. Rev. Lett. 88, 115501 (2002).

[29] L. Udby, J. Larsen, N. B. Christensen, M. Boehm, C. Niedermayer, H. E. Mohottala, T. B. S. Jensen, R. ToftPetersen, F. C. Chou, N. H. Andersen, K. Lefmann, and B. O. Wells, Phys. Rev. Lett. 111, 227001 (2013).

[30] K. Hirota, Phys. C (Amsterdam, Neth.) 357-360, 61 (2001).

[31] M. H. Julien, Phys. B (Amsterdam, Neth.) 329-333, 693 (2003).

[32] Y. S. Lee, F. C. Chou, A. Tewary, M. A. Kastner, S. H. Lee, and R. J. Birgeneau, Phys. Rev. B 69, 020502(R) (2004).

[33] S. Holm-Dahlin, J. Larsen, H. Jacobsen, A. T. Rømer, A.-E. Ţuţueanu, M. Ahmad, J.-C. Grivel, T. Goko, R. Scheuermann, M. v Zimmermann, M. Boehm, P. Steffens, K. Conder, C. Niedermayer, K. S. Pedersen, N. B. Christensen, S. B. Emery, B. O. Wells, K. Lefmann, and L. Udby (unpublished).

[34] See Supplemental Material at http://link.aps.org/supplemental/ 10.1103/PhysRevB.101.100504 for details about the samples and neutron scattering experiment. This material includes Refs. [48-57].

[35] T. Tejsner, M. Boehm, A. Piovano, A.-E. Ţuţueanu, and L. Udby (2018), LSCO+O Cu-O bond-stretching phonons. Institut Laue-Langevin (ILL), doi:10.5291/ILL-DATA.7-01-458.

[36] T. Tejsner, M. Boehm, A. Piovano, A.-E. Ţuţueanu, and L. Udby (2018), Investigation of the influence of field-induced phase-separation in $\mathrm{LSCO}+\mathrm{O}$ on the $\mathrm{Cu}-\mathrm{O}$ bond stretching phonon anomaly and spin fluctuations. Institut Laue-Langevin (ILL), doi:10.5291/ILL-DATA.7-01-474.

[37] B. Fåk and B. Dorner, Phys. B (Amsterdam, Neth.) 234-236, 1107 (1997).

[38] F. Giustino, M. L. Cohen, and S. G. Louie, Nature (London) 452, 975 (2008).

[39] D. Reznik, Phys. C (Amsterdam, Neth.) 481, 75 (2012).

[40] D. Reznik, T. Fukuda, D. Lamago, A. Q. R. Baron, S. Tsutsui, M. Fujita, and K. Yamada, J. Phys. Chem. Solids 69, 3103 (2008).

[41] S. R. Park, Y. Cao, Q. Wang, M. Fujita, K. Yamada, S.-K. Mo, D. S. Dessau, and D. Reznik, Phys. Rev. B 88, 220503(R) (2013).

[42] D. R. Garcia and A. Lanzara, Adv. Condens. Matter Phys. 2010 (2010).

[43] Z. Zhang, R. Sutarto, F. He, F. C. Chou, L. Udby, S. L. Holm, Z. H. Zhu, W. A. Hines, J. I. Budnick, and B. O. Wells, Phys. Rev. Lett. 121, 067602 (2018).

[44] T. Tejsner, S. Holm-Dahlin, A. T. Rømer, H. Jacobsen, M. Boehm, A. Piovano, and L. Udby (unpublished).

[45] D. Reznik, D. Parshall, S. R. Park, J. W. Lynn, and T. Wolf, J. Supercond. Novel Magn. 29, 643 (2016).

[46] M. Fujita, H. Goka, K. Yamada, J. M. Tranquada, and L. P. Regnault, Phys. Rev. B 70, 104517 (2004). 
[47] S. A. Kivelson, E. Fradkin, and V. J. Emery, Nature (London) 393, 550 (1998).

[48] H. Jacobsen, S. L. Holm, M.-E. Lăcătuşu, A. T. Rømer, M. Bertelsen, M. Boehm, R. Toft-Petersen, J.-C. Grivel, S. B. Emery, L. Udby, B. O. Wells, and K. Lefmann, Phys. Rev. Lett. 120, 037003 (2018).

[49] R. J. McQueeney, Y. Petrov, T. Egami, M. Yethiraj, G. Shirane, and Y. Endoh, Phys. Rev. Lett. 82, 628 (1999).

[50] G. Shirane, S. M. Shapiro, and J. M. Tranquada, Neutron Scattering with a Triple-Axis Spectrometer: Basic Techniques (Cambridge University Press, Cambridge, UK, 2002).
[51] G. Kresse and J. Hafner, Phys. Rev. B 48, 13115 (1993).

[52] G. Kresse and J. Furthmüller, Comput. Mater. Sci. 6, 15 (1996).

[53] G. Kresse and J. Furthmüller, Phys. Rev. B 54, 11169 (1996).

[54] G. Kresse and D. Joubert, Phys. Rev. B 59, 1758 (1999).

[55] G. I. Csonka, J. P. Perdew, A. Ruzsinszky, P. H. T. Philipsen, S. Lebègue, J. Paier, O. A. Vydrov, and J. G. Ángyán, Phys. Rev. B 79, 155107 (2009).

[56] A. Togo and I. Tanaka, Scr. Mater. 108, 1 (2015).

[57] J. Šaroun and J. Kulda, Phys. B (Amsterdam, Neth.) 234-236, 1102 (1997). 